\title{
La Société glaronnaise de médecine
}

\section{Liana Gerber, présidente}

Correspondance: PD Dr Liana Gerber Schweizerhofstrasse 3 CH-8750 Glarus

liana.gerber@hin.ch www.glaeg.ch

\section{Le début: un cercle médico-chirurgical}

Fondée en 1834, la Société glaronnaise de médecine a fêté ses 175 ans cette année et a, pour la première fois de son histoire, nommé une femme à sa tête. A l'occasion de cet anniversaire, ses membres jettent un regard rétrospectif sur une histoire riche en traditions tout en entreprenant des changements novateurs.

L'origine de notre société se trouve au sein d'un cercle médico-chirurgical, né en son temps du besoin d'échange et de collaboration entre médecins et chirurgiens. Ce n'est que plus tard qu'a été adopté le nom de «Société cantonale médico-chirurgicale». Ni assurances ni systèmes tarifaires et par conséquent aucune contrainte économique n'existaient encore à l'époque, les questions de politique professionnelle ne figuraient donc pas à l'ordre du jour. Ce n'est qu'autour de 1900, après la création des caisses maladie, que les médecins ont ressenti le besoin de défendre ensemble leurs intérêts. De ce fait, la société qui en 1934 ne regroupait qu'une douzaine de membres en compte 97 aujourd'hui.

\section{Les tâches diverses d'aujourd'hui}

La désignation actuelle de la société date de 1974. Alors que les échanges et la bonne collaboration sont toujours à l'ordre du jour, les intérêts et les activités dans le cadre de la société se sont considérablement ouverts à d'autres domaines. La formation postgraduée de nos membres et une solide formation continue de la relève font partie de nos principales priorités. A l'instar d'autres sociétés cantonales, la politique professionnelle est au centre de nos préoccupations et c'est pourquoi notre société siège dans des organisations faîtières plus fortes (Chambre médicale, Conférence des sociétés cantonales de médecine [CCM], Association des sociétés médicales de Suisse alémanique [VEDAG], Conférence des sociétés médicales de Suisse orientale $[\mathrm{K}-\mathrm{OCH}]$ ).

Notre société partage l'avis d'autres associations et pense que la politique actuelle de l'OFSP en matière de santé est en passe de détruire le système helvétique du médecin de famille qui, pourtant, fonctionne bien. Cette dégradation engendrera une augmentation des coûts médicaux et une baisse de la qualité tant dans le système de santé en général qu'en termes de soin. C'est pourquoi la petite société que nous sommes se rallie aux mouvements des médecins sur le plan national (p.ex. les protestations contre l'introduction de la nouvelle Liste des analyses et de la taxe de consultation, la levée de boucliers contre la suppression de la propharmacie et la lutte pour des tarifs équitables).

De plus, nous proposons des idées concrètes et des mesures visant à soutenir nos membres dans leur activité médicale ou à simplifier leur tâche, notamment en appuyant un projet de centre d'appels commun, mis en place pour les membres de la Conférence des sociétés médicales de Suisse orientale. Pour lutter contre l'explosion des coûts dans le domaine de la santé, nous voulons promouvoir l'information des patients, de même qu'une attitude positive envers les mesures de prévention. C'est pourquoi, à l'occasion de nos 175 ans, les membres de notre comité ont organisé toute une série de conférences publiques sur différents sujets: l'obésité, les facteurs de risques coronariens, les nouveautés en orthopédie et sur la vessie irritable.

La société est également très active à l'interne. Notre site internet (www.glaeg.ch) ne sert pas uniquement à nous présenter, mais également à diffuser des informations utiles sur les sujets d'actualité, telles la grippe $\mathrm{A}(\mathrm{H} 1 \mathrm{~N} 1)$, la liste de produits dopants, les directives relatives à la Loi sur les stupéfiants, des informations relatives à la formation continue etc. Des forums de discussions sont également à disposition de nos membres. En pleine ère informatique, nos membres gardent intacte l'envie d'échanger de vive voix leurs vues entre collègues et de se soutenir mutuellement. 